\title{
ROLLING CONTACT BETWEEN DISSIMILAR VISCOELASTIC CYLINDERS*
}

\author{
L. W. MORLAND \\ University of East Anglia
}

Summary. This paper treats the plane problem of rolling contact between linear viscoelastic cylinders with different radii and different quantitative mechanical response. The analysis is an extension of that previously given for the simpler problem of rolling contact between two identical cylinders (or equivalently one cylinder and a rigid halfplane), for which a singular integral equation was derived connecting pressure and normal displacement in the contact region. The present problem is shown to lead to an integral equation of identical form but containing further parameters which reflect the difference in the properties of the two cylinders. A neater construction of the closed form solution of the integral equation is presented and the final formulae are expressed in terms of tabulated functions. An illustration is given for a viscoelastic model with two characteristic times.

1. Introduction. In many technical applications, contact between moving parts is designed to be smooth, commonly by lubrication. The effect is to reduce the shear traction in the contact region to a level small in comparison with the normal pressure, so that a first approximation is the complete neglect of shear traction. In this idealised situation the relative tangential motion of the surfaces is not restricted. The net resisting traction and couple acting on each contact body are now resultants of the normal pressure distribution over the contact region.

Plane surfaces and circular cylinders provide the simplest contact geometries for an investigation of the pressure distribution and resulting resistance to motion, and are commonly adopted in experimental testing devices. A typical situation is contact between rotating cylinders, which is treated in this paper on the further simplifying assumptions of plane deformation, constant angular velocity for each cylinder, and neglect of inertia effects. The latter include both the linear acceleration and the centrifugal force which arises with respect to axes fixed in a rotating cylinder, so that small angular velocity in both senses is implied. The elastic analysis of the problem leads to a symmetric contact pressure distribution and consequently no net traction or couple. Nonsymmetric pressure and the resulting resistance to the motion are associated with inelastic behavior of one or both contact bodies. In this paper the problem is analysed with both cylinders exhibiting linear viscoelastic behaviour but each with a different quantitative response. The angular velocities of the two cylinders are related by a rolling (nonslip) condition in mean, but in view of the assumed lack of restriction on the relative tangential velocity any prescribed angular velocities are consistent with the boundary conditions.

A detailed treatment for the case of identical viscoelastic cylinders has been given in [1], and there the symmetry leads to a plane contact region so that the pressure solution applies also to a viscoelastic cylinder rolling over a rigid plane, and, in fact, is shown also to apply to a rigid cylinder rolling over a viscoelastic half-plane. The present extension to dissimilar cylinders involves a more general contact region shape, not prescribed but a part of the required solution. This new unknown function enters

*Received September 6, 1966; revised manuscript received December 29, 1966. 
the individual boundary displacements of the two cylinders and therefore complicates the separate boundary conditions for each cylinder. But the contact geometry provides an expression for a particular additive combination of the normal displacements of the two cylinders, independent of the contact shape, and forming the corresponding combination of integral representations for the individual cylinder displacements, derived in [1], leads to a singular integral equation for the contact pressure. These integral representations and resulting integral kernel depend explicitly on the viscoelastic model adopted, which also introduces parameters relevant to further discussion of the equations and solution. It is therefore helpful at this point to describe the qualitative behaviour assumed for both cylinders.

An isotropic linear viscoelastic material may be defined in terms of two creep functions, for example a shear response $J(t)$ and dilation response $\chi(t)$. It was shown in [1] that there is no loss in generality of the integral representations, qualitative or mathematical, by assuming incompressibility, that is, setting $\chi(t) \equiv 0$, and this algebraic simplification is adopted here. The simplified stress-strain laws become

$$
\boldsymbol{\epsilon}_{k k} \equiv 0, \quad e_{i j}(\mathbf{x}, t)=J_{0} s_{i j}(\mathbf{x}, t)+\int_{-\infty}^{t} J^{\prime}\left(t-t^{\prime}\right) s_{i j}\left(\mathbf{x}, t^{\prime}\right) d t^{\prime},
$$

where $\epsilon_{k k}$ is the dilatation, and $s_{i i}, e_{i j}$ are the usual physical components of the deviatoric stress and strain tensors respectively. $\mathbf{x}$ denotes position with respect to fixed axes and $t$ is the current time. $J^{\prime}(t)$, where ' denotes derivative with respect to argument, is understood to be zero for $t \leq 0$. The response is now governed by the single creep function $J(t)$, for which a model with a finite spectrum of characteristic times $\tau_{r}(r=1, \cdots, N)$ is assumed, namely

$$
J(t)=J_{0}\left\{1+\sum_{r=1}^{N} j_{r}\left(1-\exp \left[-t / \tau_{r}\right]\right)\right\} H(t) .
$$

$H(t)$ is the Heaviside step function, $J_{0}$ is the instantaneous (elastic) response, and $j_{r}(r=1, \cdots N)$ are the weightings associated with the characteristic times. The long time (equilibrium) response is $J_{0}\left\{1+\sum_{r=1}^{N} j_{r}\right\}$.

To distinguish quantities associated with the individual cylinders, superior - , respectively will be used throughout. Thus the viscoelastic response function (1.2) for one cylinder will be defined by a set of parameters $\bar{J}_{0}, \bar{j}_{r}, \bar{\tau}_{r}(r=1, \cdots, M)$, and for the second cylinder by a set $\hat{J}_{0}, \hat{\jmath}_{r}, \hat{\tau}_{r}(r=1, \cdots, N)$. That is, models with $M$ and $N$ characteristic times respectively.

Now the displacement representation for an individual cylinder is comprised of a term associated with the instantaneous response, and a sum of similar terms each associated with a characteristic time. On forming the additive combination of the two displacements arising in the contact conditions a sum of similar terms is obtained corresponding to a total of $M+N$ characteristic times. In turn the resulting integral equation has the same form as that for an identical cylinder problem in which the common creep function has a spectrum of $M+N$ characteristic times. Thus the dissimilar cylinders problem is reduced to an identical cylinders problem with a common creep function containing the composite number of characteristic times. The actual reduced times, their weightings, and the instantaneous response, depend generally on the sets of parameters of both cylinders, but there is some flexibility in relating these reduced properties to those of the two cylinders. It is found convenient for comparison of the two problems to match the reduced angular velocity, radius, and 
instantaneous response, with those of one cylinder, while retaining the ratios of all $M+N$ characteristic times to contact time and prescribing the same total normal force (along the line of centres). Then the contact angles, contact time, and the actual characteristic times and weightings occurring in the two problems differ in a determined manner.

It remains to comment on the common integral equation and its solution. For convenience the reduced creep function will be described by (1.2), the model with $N$ characteristic times. The solution given in [1] involved the construction of an $N$ th order differential equation for the finite Hilbert transform of the pressure, but with no simple formulae for the (constant) coefficients. A closed form inversion for the typical exponential term was obtained but the $N$ integration constants depended on integrals requiring numerical evaluation. In the present treatment it is shown that by adopting the known form of the Hilbert transform, its complete specification can be derived directly from the original integral equation in terms of simple algebraic equations. In addition, the analysis is extended to evaluate the one remaining integral in terms of tabulated functions. A considerable shortening of the numerical calculations needed to present a quantitative solution is thus obtained.

Effective measures of the nonsymmetry arising in the pressure distribution are provided by two parameters. These are $\Delta$, a fractional angular displacement of the central contact radius from the line of centres, and $\mu$, a coefficient of rolling friction defined as the ratio of transverse to normal force divided by the semicontact angle. The latter takes into account that the transverse force is necessarily smaller than the normal force by a factor of order the contact angle, since it is a resolution of normal pressure only. Both $\Delta$ and $\mu$ depend on the ratios of the characteristic times to contact time and their weightings relative to the instantancous response. In order to exhibit the interplay of these variables a detailed numerical illustration is presented for a model with two characteristic times, equivalent to dissimilar cylinders each represented by a single-time model, for which there are four such variables.

2. Contact conditions. Figure 1 illustrates the contact geometry with the contact boundary $A B$ greatly enlarged; the curve $A B$ is unknown. The superior quantities associated with the respective cylinders. $\bar{\epsilon}, \hat{\epsilon}$ are the respective semicontact angles, and the bisectors of the contact angles, $\bar{O} C, \hat{O} C$ make angles $\bar{\delta}$, $\delta$ with the line of centres $\bar{O} \hat{O} ; \bar{\delta}, \hat{\delta}$ are to be determined in the solution. $\bar{O} C$ and $\hat{O} C$ are used as the fixed zero lines for the polar angles $\bar{\theta}, \hat{\theta}$ in the cylinders of radii $\bar{R}, \hat{R}$ respectively, measured positive in the sense of the angular velocities $\bar{\omega}, \hat{\omega}$. $P$ is a general point on the contact boundary with polar coordinates $(\bar{r}, \bar{\theta}),(\hat{r}, \hat{\theta}), \hat{\delta})$ respectively. $\bar{d}$, $\hat{d}$ are nondimensional measures of the indentation depths. The viscoelastic stress relaxation is responsible for the shift of the central lines $\bar{O} C, \hat{O} C$ away from the line of centres $\bar{O} \hat{O}$ and towards the leading edge $A$ of the contact region.

Now it was shown in [1] that the semicontact angle is a measure of the maximum strain magnitude occurring in the identical cylinders, so that within the linear theory terms of order $\epsilon$ are neglected in comparison with unity. If pressures and (or) materials are such that the contact angle cannot be regarded as infinitesimal, then the above implies that the nonlinear theory must be used. On applying this linearisation in each cylinder, that is neglecting terms of order $\bar{\epsilon}$, $\hat{\epsilon}$ respectively, which will be discussed in Sec. 4 , it is easily seen that

$$
\hat{\epsilon} / \bar{\epsilon}=\delta / \bar{\delta}=\hat{\theta} / \bar{\theta}=\bar{r} / \hat{r}=\bar{R} / \hat{R}=k,
$$




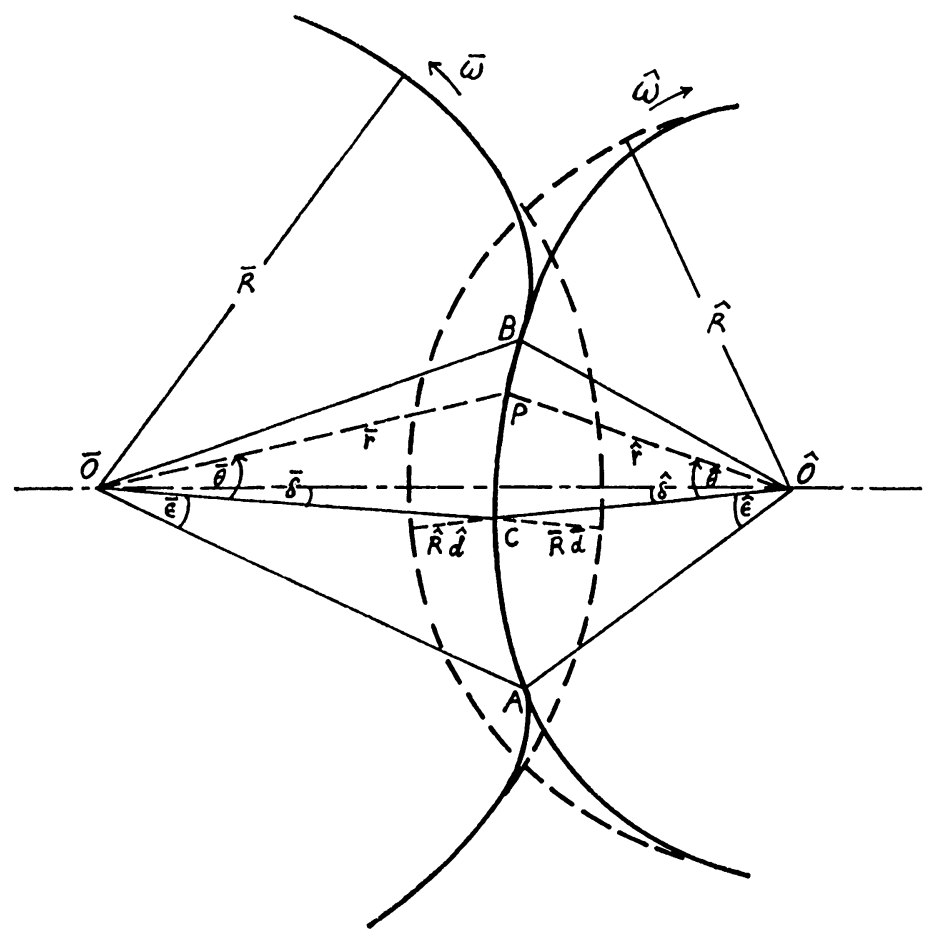

FIG. 1. Contact geometry.

where the ratio $k$ is a useful geometric parameter. Further, with the assumption of rolling contact in mean, an overall nonslip condition, the mean circumferential velocities are the same and the angular velocities satisfy

$$
\hat{\omega} / \ddot{\omega}=k .
$$

The current neglect of shear traction over the contact region allows, if required, independent prescription of $\bar{\omega}, \hat{\omega}$.

The respective coordinates of the point $P$ on the contact boundary are related by the geometric identity

$$
\bar{r} \cos (\bar{\theta}-\bar{\delta})+\hat{r} \cos (\hat{\theta}-\hat{\delta})=\bar{R}(1-\bar{d}) \cos \bar{\delta}+\hat{R}(1-\hat{d}) \cos \hat{\delta} .
$$

With the definition of nondimensional angular displacements

$$
x=\bar{\theta} / \bar{\epsilon}=\hat{\theta} / \hat{\epsilon}, \quad \Delta=\bar{\delta} / \bar{\epsilon}=\hat{\delta} / \hat{\epsilon},
$$

it follows from (2.3) that

$$
\frac{\bar{r}}{\bar{R}}+\frac{1}{k} \frac{\hat{r}}{\hat{R}}=1-\bar{d}+\frac{1-\hat{d}}{k}+\frac{1}{2} \bar{\epsilon}^{2}(1+k)\left(x^{2}-2 \Delta x\right)+o\left(\epsilon^{3}\right), \quad|x| \leq 1,
$$

where $\epsilon$ represents max $(\bar{\epsilon}, \hat{\epsilon})$. Here the previous result $d=O\left(\epsilon^{2} \log \epsilon\right)$ has been used. It will be seen in Sec. 3 that it is the $x$-dependent term of order $\bar{\epsilon}^{2}$ that is significant.

The displacement of the particle (in each cylinder) currently at the point $P$ on the contact boundary involves both radial and transverse components, and the contact 
geometry provides only a relation for a combination of these two components (for both cylinders). It was shown in [1] that the transverse component term makes no first order contribution so that within the linear theory the displacement is described by a radial component $u$. Then for the two cylinders,

$$
\bar{u} / \bar{R}=\bar{r} / \bar{R}-1, \quad \hat{u} / \hat{R}=\hat{r} / \hat{R}-1,
$$

where $\bar{u}, \hat{u}$ are the displacements at $P$. Thus (2.5) becomes

$$
\frac{\bar{u}}{\bar{R}}+\frac{1}{k} \frac{\hat{u}}{\hat{R}}=-\bar{d}-\frac{\hat{d}}{k}+\frac{1}{2} \bar{\epsilon}^{2}(1+k)\left(x^{2}-2 \Delta x\right), \quad|x| \leq 1,
$$

anticipating the removal of the higher order terms, which gives an expression for a combination of the individual cylinder displacements at a general contact point $P$.

For each cylinder the stress boundary conditions are simply zero shear traction over the entire circumference, zero normal (radial) traction over the circumference outside the contact region, and within the contact region normal pressures $\bar{p}(\bar{\theta}), \hat{p}(\hat{\theta})$. Continuity of pressure at the point $P$ implies

$$
\bar{p}(\bar{\epsilon} x)=\hat{p}(\hat{\epsilon} x), \quad|x| \leq 1,
$$

where it is assumed that the pressure is bounded at the end points $x= \pm 1$ in view of the smooth contact. It is convenient for subsequent application of integral representations derived in [1] to introduce dimensionless pressures for the individual cylinders by the relations

$$
\bar{J}_{0} \bar{p}(\bar{\theta})=\bar{\epsilon} \bar{P}(x), \quad \hat{J}_{0} \hat{p}(\hat{\theta})=\hat{\epsilon} \hat{P}(x),
$$

when the continuity condition (2.S) becomes

$$
\hat{J}_{0} \bar{P}(x)=k \bar{J}_{0} \hat{P}(x), \quad|x| \leq 1 .
$$

The result that the maximum strain in each cylinder is of the order of magnitude of the contact angle rests on showing that $\bar{P}(x), \hat{P}(x)$ are of order unity, which will be discussed in Sec. 4.

The contact tractions on each cylinder are assumed to be balanced by line forces on the cylinder axis, which, as shown in [1], make no first order contribution to the boundary displacements. Using the convention that the normal pressure is applied to the undeformed circular boundary, no balancing couple at the centre is required. Let $T$ denote the normal (line of centres) resultant of the pressure distribution and $S$ the transverse resultant (opposing the motion), respectively equal for the two cylinders. Then in terms of the dimensionless pressures,

$$
\begin{gathered}
\bar{T}=\hat{T}=\frac{\bar{\epsilon}^{2} \bar{R}}{\bar{J}_{0}} \int_{-1}^{1} \bar{P}(x) d x=\frac{\hat{\epsilon}^{2} \hat{R}}{\hat{J}_{0}} \int_{-1}^{1} \hat{P}(x) d x, \\
\bar{S}=\hat{S}=\Delta \bar{\epsilon} \bar{T}-\frac{\bar{\epsilon}^{3} \bar{R}}{\bar{J}_{0}} \int_{-1}^{1} x \bar{P}(x) d x=\Delta \hat{\epsilon}^{\hat{T}}-\frac{\hat{\epsilon}^{3} \hat{R}}{\hat{J}_{0}} \int_{-1}^{1} x \hat{P}(x) d x,
\end{gathered}
$$

within the linear approximation. The additional contact angle factor in $S$ is indicated in this form. A coefficient of rolling friction is now defined as

$$
\bar{\mu}=\hat{\mu}=\bar{S} / \bar{\epsilon} \bar{T}=\hat{S} / \hat{\epsilon} \hat{T}=\Delta-\int_{-1}^{1} x \bar{P}(x) d x / \int_{-1}^{1} \bar{P}(x) d x,
$$

omitting the alternative expression. This provides a measure of the mechanical effect of the nonsymmetry in the pressure distribution while $\Delta$ represents the nonsymmetry of the contact boundary. 
3. An integral equation formulation. It is proposed to start from the integral representation derived in [1] for the boundary displacement of a rotating viscoelastic cylinder. This was obtained with the same boundary traction conditions as apply to each cylinder here, namely zero shear traction everywhere and normal pressure over a contact region subtending by a small contact angle, and with the assumption of steady state with respect to the (nonrotating) polar coordinates $(r, \theta)$. Inertia terms are neglected and all known second order terms in the contact angle are eliminated. Then for the viscoelastic model (1.2) with $N$ characteristic times, and in the notation defined in the last section, this representation is

$\frac{d}{d x}\left(-\frac{u}{\epsilon^{2} R}\right)=\frac{1}{\pi} \int_{-1}^{1} P\left(x^{\prime}\right)\left[\frac{1}{x^{\prime}-x}-\sum_{r=1}^{N} \gamma_{r}\left(\alpha_{r}-1\right) V_{r}\left(x-x^{\prime}\right)\right] d x^{\prime}, \quad|x|<1$,

applying only to the contact region. The parameters $\gamma_{r}$ and $\alpha_{r}$ are defined by

$$
\gamma_{r}=\epsilon / \omega \tau_{r}, \quad \alpha_{r}=1+j_{r} \gamma_{r}^{2} /\left(\epsilon^{2}+\gamma_{r}^{2}\right) \quad(r=1, \cdots, N),
$$

while the kernel functions $V_{r}(x)$ are given by

$$
V_{r}(x)=-\exp \left[-\gamma_{r} x\right] \int_{-\gamma r x}^{\infty} \frac{e^{-s}}{s} d s \quad(r=1, \cdots, N) .
$$

The $\gamma_{r}$ are ratios of semicontact time to each characteristic time, and so contain the dependence on the angular velocity $\omega$. For $\gamma_{r}$ of order unity (or greater), which excludes some range of large angular velocity, $\alpha_{r}=1+j_{r}$ to the first order and the factor $\left(\alpha_{r}-1\right)$ is simply the weighting $j_{r}$ associated with $\tau_{r}$. It is assumed that each weighting $j_{r}$ is of order unity so that only creep terms comparable with the instantaneous response are retained in the model.

Each $V_{r}(x)$ has a logarithmic singularity at $x=0$, while the leading term of the kernel in (3.1) is a strong singularity with the integral interpreted as a Cauchy principal value, requiring continuity of $P(x)$ in $|x|<1$. By introducing the new functions

$$
\mathscr{g}(x)=\frac{1}{\pi} \int_{-1}^{1} \frac{P\left(x^{\prime}\right) d x^{\prime}}{x^{\prime}-x}, \quad \mathscr{G}_{r}(x)=-\frac{1}{\pi} \int_{-1}^{1} P\left(x^{\prime}\right) V_{r}\left(x-x^{\prime}\right) d x^{\prime}, \quad(r=1, \cdots, N)
$$

where $\mathfrak{g}(x)$ is the finite Hilbert transform of $P(x)$ \{see, for example, [2, p. 173]\}, (3.1) can be rewritten

$$
\frac{d}{d x}\left(-\frac{u}{\epsilon^{2} R}\right)=\mathfrak{g}(x)+\sum_{r=1}^{N} \gamma_{r}\left(\alpha_{r}-1\right) \mathscr{I}_{r}(x), \quad|x|<1 .
$$

The following properties will be used in Sec. 5. The functions $\mathscr{S}(x), \mathfrak{g}_{r}(x)$ are defined by (3.4) for all $x$, and in particular are continuous at $x= \pm 1$ for bounded $P(x)$. Differentiating $\mathscr{I}_{r}(x)$, and using (3.3), shows that

$$
\mathfrak{I}_{r}^{\prime}(x)+\gamma_{r} \mathscr{G}_{r}(x)=\mathscr{I}(x) ; \quad|x| \neq 1, \quad(r=1, \cdots, N),
$$

where $\mathcal{S}_{r}^{\prime}(x)$ is not nceessarily defined at $x= \pm 1$. Further from (3.4) and (3.3),

$$
\mathscr{I}(x), \mathscr{I}_{r}(x)=O(1 /|x|) \quad \text { as } \quad|x| \rightarrow \infty \quad(r=1, \cdots, N) .
$$

The above results may now be applied to each cylinder separately, taking viscoelastic models with $M$ and $N$ characteristic times $\bar{\tau}_{r}, \hat{\tau}_{r}$ respectively. Let $J$ be the ratio of the instantaneous creep responses of the two cylinders defined by

$$
\hat{J}_{0}=J \bar{J}_{0} \text {, }
$$


then the continuity condition (2.10) and definition (3.4) give

$$
k \hat{P}(x)=J \bar{P}(x), \quad k \hat{\mathfrak{I}}(x)=J \bar{J}(x) .
$$

Differentiating (2.7) and substituting the representations (3.5) for each cylinder, using the second relation (3.9), yields

$$
\begin{array}{r}
(1+J) \overline{\mathscr{S}}(x)+\sum_{r=1}^{M} \bar{\gamma}_{r}\left(\bar{\alpha}_{r}-1\right) \overline{\mathfrak{I}}_{r}(x)+k \sum_{r=1}^{N} \hat{\gamma}_{r}\left(\hat{\alpha}_{r}-1\right) \hat{\mathfrak{I}}_{r}(x)=(1+k)(\Delta-x), \\
|x|<1,
\end{array}
$$

where the indentation depths $\bar{d}, \hat{d}$ are now eliminated. Using the pressure identity in (3.9) with the definition (3.4) expresses $\hat{\mathscr{g}}_{r}(x)$ in terms of $\bar{P}(x)$, each $r=1, \cdots, N$, so that (3.10) then constitutes an integral equation for $\bar{P}(x)$. Alternatively it may be rewritten as an integral equation for $\hat{P}(x)$.

In fact (3.10) can be reduced to an integral equation for an identical cylinders problem. Defining a new dimensionless pressure $P(x)$ by

$$
P(x)=\frac{1+J}{1+k} \bar{P}(x), \quad=\frac{k}{J} \cdot \frac{1+J}{1+k} \hat{P}(x),
$$

and a set of parameters $\gamma_{r}, \alpha_{r}(r=1, \cdots, M+N)$ by

$$
\begin{aligned}
\gamma_{r} & =\bar{\gamma}_{r} \quad(r=1, \cdots, M), \\
& =\hat{\gamma}_{r-M} \quad(r=M+1, \cdots, M+N), \\
\alpha_{r}-1 & =\frac{\bar{\alpha}_{r}-1}{1+J} \quad(r=1, \cdots, M), \\
& =J \frac{\hat{\alpha}_{r-M}-1}{1+J} \quad(r=M+1, \cdots, M+N),
\end{aligned}
$$

together with the associated functions $\mathscr{I}(x), V_{r}(x), \mathscr{I}_{r}(x)(r=1, \cdots, M+N)$ as given in (3.3), (3.4), allows (3.10) to be rewritten as

$$
\mathfrak{I}(x)+\sum_{r=1}^{M+N} \gamma_{r}\left(\alpha_{r}-1\right) \mathscr{I}_{r}(x)=\Delta-x, \quad|x|<1 .
$$

Since $V_{r}(x)$ is independent of $\alpha_{r}$, and depends only on $\gamma_{r}$ which by (3.2) is one of the $\bar{\gamma}_{r}$ or $\hat{\gamma}_{r}$, each $V_{r}(x)(r=1, \cdots, M+N)$ is one of the $\bar{V}_{r}(x)$ or $\hat{V}_{r}(x)$ and the differential relation (3.6) between $\mathscr{I}_{r}(x), \mathfrak{I}(x)$ holds for each $r=1, \cdots, M+N$. Also the asymptotic properties (3.7) apply. The integral equation (3.13) is that derived in [1] for the dimensionless pressure $P(x)$ of an identical cylinders problem with the single set of parameters $\gamma_{r}, \alpha_{r}(r=1, \cdots, M+N)$, corresponding to a common viscoelastic creep function with $M+N$ characteristic times $\tau_{r}$. The semicontact angle $\epsilon$, angular velocity $\omega$, and weightings $j_{r}(r=1, \cdots, M+N)$ are given by the relations (3.2). Further, the R.H.S. of (3.13) shows that $\Delta$ is also the off-centre shift of the contact region in the reduced identical cylinders problem.

4. Comparison of equations for dissimilar and identical cylinders. The introduction of new quantities by (3.11), (3.12) reduces the dissimilar cylinders problem to the integral equation (3.13) for an identical cylinders problem, and (3.13) can now be regarded as the standard equation for all cases. A corresponding standard solution will be obtained in closed form in Sec. 5, but first we will examine the class of dissimilar cylinder solutions generated by a solution of (3.13), that is for specified values of $\gamma_{r}, \alpha_{r}(r=1, \cdots, M+N)$. 
Recall that the contact region shift $\Delta$ is not prescribed, but is part of the solution, and being unchanged in the reduction is therefore determined before the parameters reflecting the different properties of the two cylinders are chosen. By (3.12) the $\bar{\gamma}_{r}, \hat{\gamma}_{r}$ are fixed, which, from (3.2), determines the ratios of the $\bar{\tau}_{r}$ and of the $\hat{\tau}_{r}$, but not their magnitudes until $\bar{\epsilon} / \bar{\omega}(=\hat{\epsilon} / \hat{\omega})$ is chosen. Also the $\bar{\alpha}_{r}$ and $\hat{\alpha}_{r}$ are not fixed until $J$ is chosen. The actual contact pressure distribution $p(\theta)$ in the two problems depends, through (2.9), on the respective dimensionless pressure, contact angle, and instantaneous response of the material, and the corresponding resultant normal forces, from (2.11) and (3.11), satisfy

$$
\bar{T}=\hat{T}=\frac{1+k}{1+J} \cdot \frac{\bar{\epsilon}^{2}}{\epsilon} \cdot \frac{\bar{R}}{R} \cdot \frac{J_{0}}{\bar{J}_{0}} T,
$$

where $\epsilon, R, J_{0}, T$ refer to the reduced identical cylinders problem. Similarly, from (2.13) and (3.11), the reduced coefficient of rolling friction, $\mu$, satisfies

and so is unchanged in the reduction.

$$
\bar{\mu}=\hat{\mu}=\mu,
$$

For a comparison of the two problems it is convenient to identify the reduced common cylinder properties with the ${ }^{-}$cylinder (say) properties to the extent

$$
J_{0}=\bar{J}_{0}, \quad R=\bar{R}_{0}, \quad \omega=\bar{\omega},
$$

when (3.12) further implies

$$
\bar{\epsilon} \tau_{r}=\epsilon \begin{cases}\bar{\tau}_{r} & (r=1, \cdots, M) \\ \hat{\tau}_{r-M} & (r=M+1, \cdots, M+N) .\end{cases}
$$

Now, either the normal forces or semicontact angles may be matched, with the implications

$$
T=\bar{T} \Rightarrow \bar{\epsilon}^{2}=\frac{1+J}{1+k} \epsilon^{2}, \quad \epsilon=\bar{\epsilon} \Rightarrow \bar{T}=\frac{1+k}{1+J} T .
$$

In both cases the characteristic times $\bar{\tau}_{r}, \hat{\tau}_{r}$ are then related to the $\tau_{r}$ by (4.4). If the two cylinders are in fact identical, when $k=1, \bar{\epsilon}=\hat{\epsilon}, J=1, M=N, \ddot{\tau}_{r}=\hat{\tau}_{r}, \bar{\alpha}_{r}=\hat{\alpha}_{r}$ $(r=1, \cdots, N)$, it follows trivially that $T=\bar{T}$ and $\alpha_{r}-1=\alpha_{N+r}-1=\frac{1}{2}\left(\bar{\alpha}_{r}-1\right)$, $\tau_{r}=\tau_{N+r}=\bar{\tau}_{r}(r=1, \cdots, N)$. That is, the $2 N$ characteristic times reduce to the $N$ distinct $\bar{\tau}_{r}$ each with a total weighting $\bar{\alpha}_{r}-1$ which gives the required identical cylinders integral equation.

It remains to discuss the validity of the integral equation (3.13) as the exact (linear) formulation of the dissimilar cylinders problem. Recall that the single cylinder results taken from [1] neglect terms, in comparison with unity, which have the same magnitude as the contact angle. The justification rested on showing $P(x)$ is of order unity, so that by $(2.9) \epsilon$ and $J_{0} p(\theta)$, a measure of the strain in the contact region, are of the same order of magnitude. In application to the separate cylinders terms of order $\bar{\epsilon}, \hat{\epsilon}$ respectively are neglected, and we have yet to examine the respective magnitudes of strain. Since the solution of (3.13) shows that $P(x)$ is of order unity, the magnitudes of $\bar{P}(x)$ and $\hat{P}(x)$ are given in terms of $k$ and $J$ by (3.11), and hence the magnitudes of $\bar{J}_{0} \bar{p}(\bar{\theta})$ and $\hat{J}_{0} \hat{p}(\hat{\theta})$, the respective strain-measures, follow from (2.9). Clearly if $k$ and $J$ are separately of order unity, which is the typical case in practice, or, less restrictive, the ratio $k / J$ is of order unity, then $\bar{P}(x)$ and $\hat{P}(x)$ are of order unity and give the respectivestrain measures as required. In particular the limiting case of viscoelastic cylinder and rigid half-plane (equivalent to an identical cylinders problem) is given by $k / J=1$.

In gencral, the identification of contact angle with strain measure in each cylinder devolves on the dimensionless pressures $\bar{P}(x), \hat{P}(x)$ both being order unity, which in turn, 
from (3.11), requires both $(1+k) /(1+J)$ and $J(1+k) / k(1+J)$ to be of order unity. But the validity of neglecting terms of order $\bar{\epsilon}, \hat{\epsilon}$ within the linear theory requires only that $\bar{\epsilon}, \hat{\epsilon}$ are not greater in magnitude than the respective strain measures. Hence the less restrictive condition is that $\bar{P}(x)$ and $\hat{P}(x)$ have magnitudes of order unity or greater, corresponding to the same condition for the factors $(1+k) /(1+J)$ and $J(1+k) / k(1+J)$. In more detail, specifying the magnitudes of $k$ and $J$ separately, these validity conditions are easily shown to be equivalent to

$$
k=O\left(\epsilon^{m}\right), \quad J=O\left(\epsilon^{l}\right) ; \quad m \geq l \geq 0 \quad \text { or } \quad 0 \geq l \geq m .
$$

Thus, although the ratio of the radii and the ratio of the instantaneous responses of the two cylinders may separately have any magnitude, these magnitudes must satisfy the restrictions (4.6). If the magnitudes of $k$ and $J$ lie outside the ranges (4.6), then at least one of $\bar{\epsilon}, \hat{\epsilon}$ is greater than the strain magnitude in the respective cylinder. Provided that $\bar{\epsilon}, \hat{\epsilon} \ll 1$, the appropriate higher order terms can be retained in both the displacement boundary conditions and integral kernels, but the resulting equations are considerably more complicated. An iteration in powers of $\bar{\epsilon}, \hat{\epsilon}$ may be feasible. As remarked earlier, if $\bar{\epsilon}$ or $\hat{\epsilon}$ is not small then finite deformation occurs in the appropriate cylinder and the linear theory is not applicable.

5. Solution of the integral equation. The two-cylinder problem is now reduced to the standard singular integral equation (3.13), which describes an identical cylinders problem for a finite spectrum model with the composite number of characteristic times; for convenience this is now defined as $N$. A closed form solution has been constructed in [1] by showing that $\mathscr{g}(x)$, which is the finite Hilbert transform of $P(x)\{[2, \mathrm{p} .173]\}$, satisfies an $N$ th order differential equation with constant coefficients. This is solved for $\mathscr{G}(x)$ and the $N$ integration constants determined by evaluating (3.13) and its first $(N-1)$ derivatives at $x=0$. Inverting the transform gives $P(x)$. It will now be shown that the construction of the differential equation coefficients and subsequent evaluation of the $(N-1)$ equation derivatives can be replaced by a neat algebraic procedure if we start from the predicted general form of solution for $\mathscr{g}(x)$. Thus, calling on results from [1], $\mathfrak{g}(x)$ takes the form

$$
g(x)=B_{0}-B_{1} x+\sum_{m=1}^{N} A_{m} e^{-\lambda_{m} x}, \quad|x|<1,
$$

where it is assumed that the roots of the auxiliary equation, $\lambda_{m}$, are distinct. Coincident roots give rise to terms $x^{*} \exp \left[-\lambda_{m} x\right]$ for which the present method, as that in [1], can be trivially extended. Inversion of the finite Hilbert transform (5.1), defined by (3.4), was shown to give

$$
\begin{aligned}
P(x)=B_{1}\left(1-x^{2}\right)^{1 / 2} & +\sum_{m=1}^{N} \lambda_{m} A_{m} \exp \left[-\lambda_{m} x\right] \\
& \cdot \int_{x}^{1}\left[t I_{0}\left(\lambda_{m}\right)-I_{1}\left(\lambda_{m}\right)\right]\left(1-t^{2}\right)^{-1 / 2} \exp \left[\lambda_{m} t\right] d t, \quad|x| \leq 1,
\end{aligned}
$$

already bounded (in fact zero) at $x=1$, together with the condition for bounded (zero) $P(-1)$,

$$
B_{0}+\sum_{m=1}^{N} A_{m} I_{0}\left(\lambda_{m}\right)=0 .
$$

$I_{0}\left(\lambda_{m}\right), I_{1}\left(\lambda_{m}\right)$ are modified Bessel functions of the first kind \{see, for example, [3, p. 77]\}. We must now determine the $N$ exponents $\lambda_{m}, N$ nonzero coefficients $A_{m}$ (giving $B_{0}$ ), $B_{1}$ and $\Delta$. 
Integrating (3.6) with $\mathscr{T}(x)$ in the form (5.1) gives for each $r=1, \cdots, N$, $\mathscr{g}_{r}(x)=\frac{\gamma_{r} B_{0}+B_{1}}{\gamma_{r}^{2}}-\frac{B_{1}}{\gamma_{r}} x+\sum_{m=1}^{N} \frac{A_{m}}{\gamma_{r}-\lambda_{m}} \exp \left[-\lambda_{m} x\right]+D_{r} \exp \left[-\gamma_{r} x\right], \quad|x|<1$

where $D_{r}$ is an arbitrary constant of integration. Note that $\gamma_{r} \neq \lambda_{m}(r, m=1, \cdots N)$ since otherwise $\mathscr{G}_{r}(x)$ would include a term $x \exp \left[-\gamma_{r} x\right]$ with no balance term in (3.13) under the present assumption of distinct $\lambda_{m}$. Substituting (5.1) and (5.4) in (3.13) and balancing the respective constant, linear, $\exp \left[-\lambda_{m} x\right]$, exp $\left[-\gamma_{r} x\right]$ terms, produces the identities

$$
\begin{gathered}
B_{0}\left\{1+\sum_{r=1}^{N}\left(\alpha_{r}-1\right)\right\}+B_{1} \sum_{r=1}^{N} \frac{\alpha_{r}-1}{\gamma_{r}}=\Delta, \\
B_{1}\left\{1+\sum_{r=1}^{N}\left(\alpha_{r}-1\right)\right\}=1, \\
A_{m} \neq 0, \quad 1+\sum_{r=1}^{N} \frac{\gamma_{r}\left(\alpha_{r}-1\right)}{\gamma_{r}-\lambda_{m}}=0 \quad(m=1, \cdots, N), \\
D_{r}=0 \quad(r=1, \cdots, N) .
\end{gathered}
$$

$B_{1}$ is determined directly by (5.6) whence (5.5) and (5.3) express $\Delta$ in terms of $B_{1}$ and the $A_{m}$. Rewriting (5.7) shows that $\lambda_{m}(m=1, \cdots, N)$ are roots of the $N$ th order polynomial equation

$$
\prod_{r=1}^{N}\left(\gamma_{r}-\lambda\right)+\sum_{r=1}^{N} \gamma_{r}\left(\alpha_{r}-1\right) \prod_{s=1 ; s \neq \dot{r}}^{N}\left(\gamma_{s}-\lambda\right)=0
$$

and since $\gamma_{r}$ and $\left(\alpha_{r}-1\right)$ are strictly positive it follows directly from (5.7) that all the roots are real and

$$
\lambda_{m}>\min \left(\gamma_{r}\right),>0, \quad(m, r=1, \cdots, N) .
$$

Finally, combining (5.8) and (5.4) and evaluating at $x=-1$ gives the $N$ relations

$$
\mathcal{I}_{r}(-1)=\frac{\gamma_{r} B_{0}+\gamma_{r} B_{1}+B_{1}}{\gamma_{r}^{2}}+\sum_{m=1}^{N} \frac{A_{m}}{\gamma_{r}-\lambda_{m}} e^{\lambda_{m}} \quad(r=1, \cdots, N),
$$

where it remains only to evaluate the $\mathscr{g}_{r}(-1)$ from the Eqs. (3.4).

In choosing the contact boundary $x=-1$ [instead of $x=0$ as chosen in [1] for the numerical evaluation \} we are following the analytic approach adopted in [4] for a single time model. There the displacement on the half-space boundary outside the contact region was considered, which, together with continuity at the contact boundary and known asymptotic behaviour at infinity, sufficed to determine the integration constant. In this cylinder problem, with no physical infinity, we could interpret similarly for the stretched coordinate $x$, but more directly, bounded $P(x)$ in $|x| \leq 1$ ensures that $\mathscr{I}(x)$, $\mathfrak{g}_{r}(x)$, defined in (3.4), are continuous at $x= \pm 1$ \{hence the strain function defined in (3.5)\} while (3.6), (3.7) provide the differential relations and asymptotic behavior. Thus performing the integration and using the behavior of $\mathfrak{J}_{r}(x)$ as $x \rightarrow-\infty$, recalling that each $\gamma_{r}>0$,

$$
\mathscr{I}_{r}(-1)=\exp \left[\gamma_{r}\right] \int_{-\infty}^{-1} \exp \left[\gamma_{r} s\right] \mathfrak{I}(s) d s \quad(r=1, \cdots N),
$$

which requires $\mathscr{G}(x)$ in $x<-1$. From the definition (3.4) with $P(x)$ given by (5.2), and using some elementary Hilbert transforms,

$$
\mathfrak{I}(x)=-B_{1}\left\{x+\left(x^{2}-1\right)^{1 / 2}\right\}+\sum_{m=1}^{N} A_{m} g_{m}(x), \quad x<-1,
$$


where

$$
\begin{aligned}
g_{m}(x)=\frac{\lambda_{m}}{\pi} \int_{-1}^{1} \frac{\exp \left[-\lambda_{m} y\right]}{y-x}\left\{\int_{\nu}^{1} \frac{\left[t I_{0}\left(\lambda_{m}\right)-I_{1}\left(\lambda_{m}\right)\right] \exp \left[\lambda_{m} t\right]}{\left(1-t^{2}\right)^{1 / 2}} d t\right\} d y & \\
& (m=1, \cdots, N) .
\end{aligned}
$$

Integration by parts, noting that the inner integral vanishes at $y=-1$, shows that

$$
g_{m}^{\prime}(x)+\lambda_{m} g_{m}(x)=\lambda_{m}\left(x^{2}-1\right)^{-1 / 2}\left[I_{1}\left(\lambda_{m}\right)-x I_{0}\left(\lambda_{m}\right)\right]-\lambda_{m} I_{0}\left(\lambda_{m}\right) .
$$

By (5.14), $g_{m}(x)=0(1 /|x|)$ as $x \rightarrow-\infty$, and by (5.10), $\lambda_{m}>0$, so on further integration $g_{m}(x)=\lambda_{m} \exp \left[-\lambda_{m} x\right] \int_{-\infty}^{x}\left[I_{1}\left(\lambda_{m}\right)-t I_{0}\left(\lambda_{m}\right)\right]\left(t^{2}-1\right)^{-1 / 2} \exp \left[\lambda_{m} t\right] d t-I_{0}\left(\lambda_{m}\right)$.

Using this result to evaluate $\mathscr{S}(-1)$ verifies continuity with the $(5.1)$ expression once the $A_{m}$ are determined.

Substitution for $\mathfrak{I}(x)$ in (5.12), and using some Bessel function integral representations [3, p. 185], gives

$$
\begin{array}{r}
\mathscr{g}_{r}(-1)=\frac{B_{1}}{\gamma_{r}^{2}}\left\{\gamma_{r}+1-\gamma_{r} \exp \left[\gamma_{r}\right] K_{1}\left(\gamma_{r}\right)\right\}-\sum_{m=r}^{N} \frac{A_{m} I_{0}\left(\lambda_{m}\right)}{\gamma_{r}}+\sum_{m=1}^{N} \lambda_{m} A_{m} \exp \left[\gamma_{r}\right] G_{m r} \\
(r=1, \cdots, N),
\end{array}
$$

where

$$
\begin{array}{r}
G_{m r}=\int_{-\infty}^{-1} \exp \left[\gamma_{r} s\right]\left\{\int_{-\infty}^{*}\left[I_{1}\left(\lambda_{m}\right)-t I_{0}\left(\lambda_{m}\right)\right]\left(t^{2}-1\right)^{-1 / 2} \exp \left[-\lambda_{m}(s-t)\right] d t\right\} d s \\
(m, r=1, \cdots, N),
\end{array}
$$

and after further integration by parts and use of a Bessel function identity [3, p. 80],

$\left(\lambda_{m}-\gamma_{r}\right) G_{m r}=I_{1}\left(\lambda_{m}\right) K_{0}\left(\gamma_{r}\right)+I_{0}\left(\lambda_{m}\right) K_{1}\left(\gamma_{r}\right)-\frac{1}{\lambda_{m}} \exp \left[\lambda_{m}-\gamma_{r}\right]$

$$
(m, r=1, \cdots, N) \text {. }
$$

$K_{0}\left(\gamma_{r}\right), K_{1}\left(\gamma_{r}\right)$ are modified Bessel functions of the second kind [3, p. 78]. Hence substituting (5.17)-(5.19) in (5.11) and eliminating $B_{0}$ by (5.3) gives finally the $N$ simultaneous equations for the $A_{m}$,

$$
\sum_{m=1}^{N} \frac{\lambda_{m} A_{m}}{\lambda_{m}-\gamma_{r}}\left[I_{1}\left(\lambda_{m}\right) K_{0}\left(\gamma_{r}\right)+I_{0}\left(\lambda_{m}\right) K_{1}\left(\gamma_{r}\right)\right]=\frac{K_{1}\left(\gamma_{r}\right)}{\gamma_{r}} B_{1} \quad(r=1, \cdots, N) .
$$

The normal and transverse forces, and rolling friction coefficient, defined in (2.11)(2.13), are now obtained from the appropriate integrals of $P(x)$ given by (5.2). If this is expressed in the form

$$
P(x)=B_{1}\left(1-x^{2}\right)^{1 / 2}+\sum_{m=1}^{N} A_{m} P_{m}(x), \quad|x| \leq 1,
$$

then simple integration by parts shows that

$$
\frac{1}{\pi} \int_{-1}^{1} P_{m}(x) d x=I_{1}\left(\lambda_{m}\right), \quad \frac{1}{\pi} \int_{-1}^{1} x P_{m}(x) d x=\frac{1}{\lambda_{m}} I_{1}\left(\lambda_{m}\right)-\frac{1}{2} I_{0}\left(\lambda_{m}\right),
$$

whence

$$
\begin{gathered}
T=\frac{\epsilon^{2} R \pi}{J_{0}}\left\{\frac{1}{2} B_{1}+\sum_{m=1}^{N} A_{m} I_{1}\left(\lambda_{m}\right)\right\} \\
\mu=\Delta+\left(\sum_{m=1}^{N} A_{m}\left[\frac{1}{2} I_{0}\left(\lambda_{m}\right)-\frac{1}{\lambda_{m}} I_{1}\left(\lambda_{m}\right)\right]\right) /\left(\frac{1}{2} B_{1}+\sum_{m=1}^{N} A_{m} I_{1}\left(\lambda_{m}\right)\right) .
\end{gathered}
$$

For completeness, by (5.5) and (5.3),

$$
\Delta=B_{1} \sum_{r=1}^{N} \frac{\alpha_{r}-1}{\gamma_{r}}-\frac{1}{B_{1}} \sum_{m=1}^{N} A_{m} I_{0}\left(\lambda_{m}\right),
$$


where $B_{1}$ is defined by (5.6), and the $\lambda_{m}, A_{m}(m=1, \cdots, N)$ respectively are determined from (5.9), (5.20). In the simplest case of a single-time model $(N=1)$, necessarily an identical cylinders problem, the above equations reduce to the formulae given in [4] after modification to the different notation, and it is easily shown that $A_{1}>0 ; P_{1}(x)>0$, $P(x)>0(|x|<1), \Delta>0$. Further, the limiting cases $\gamma_{1} \rightarrow 0$ (fast rolling), $\gamma_{1} \rightarrow \infty$ (slow rolling) lead to symmetric elastic pressure distributions with the instantaneous response and long time response respectively as modulus, and $\Delta=0$. For the $N$-time model, with the $\lambda_{m}$ roots of a polynomial and the $A_{m}$ solutions of simultaneous equations, the lack of explicit formulae prohibits such straightforward deductions analytically.

6. Illustration for a viscoelastic model with two characteristic times. Setting $N=2$ in the results of Sec. 5 gives an identical cylinders solution for a common creep function with two characteristic times. In turn a class of solutions for dissimilar cylinders is generated, as described in Sec. 4, with each cylinder represented by a creep function with a single characteristic time. The case $N=2$ will now be examined in detail.

The two characteristic times $\tau_{1}, \tau_{2}$ enter through their ratios with the semi-contact time, $\gamma_{1}$ and $\gamma_{2}$, defined by (3.2). Since fast rolling has been excluded from the present treatment it is reasonable to consider $\gamma_{1}, \gamma_{2}$ of order $\epsilon^{1 / 2}$ or greater, when from (3.2) the factors $\alpha_{1}-1$ and $\alpha_{2}-1$ are replaced by the weightings $j_{1}, j_{2}$ respectively. In the excluded range it is necessary to specify $\alpha_{1}$ and $\alpha_{2}$, determine $\epsilon$ in the solution, and recover $j_{1}$ and $j_{2}$ from (3.2). It is convenient to prescribe the weightings through their ratio and sum, where the latter measures the total creep relative to the instantaneous response, that is through the parameters

$$
h=j_{2} / j_{1}, \quad f=j_{1}+j_{2} .
$$

Similarly, for the two time ratios $\gamma_{1}, \gamma_{2}$ define

$$
\gamma=\gamma_{1}, \quad \zeta=\gamma_{2} / \gamma_{1} \text {. }
$$

The instantancous response $J_{0}$, radius $R$, and normal force $T$ affect only the determination of $\epsilon$, by (5.23), once (3.13) is solved, and then occur only in the combination $T J_{0} / R$ which can be regarded as the one prescribed quantity. $A$ single solution then applies to the consistent sets of $J_{0}, R, T$. Further, explicit dependence on $T J_{0} / R$ may be eliminated by introducing an appropriate reference magnitude for the contact angle, namely the contact angle $2 \epsilon_{s}$ when the cylinders are stationary and in equilibrium under the same normal force. The latter is obtained by setting each $\gamma_{r}=\infty$ and each $A_{m}=0$ in (5.23) and (5.6), when, on using (6.1),

Then by (5.23), (5.6), and (6.1),

$$
\epsilon_{s}^{2}=2(1+f) T J_{0} / \pi R .
$$

$$
\frac{\epsilon_{s}^{2}}{\epsilon}=1+2(1+f) \sum_{m=1}^{2} A_{m} I_{1}\left(\lambda_{m}\right)
$$

where $\lambda_{1}, \lambda_{2}$ are roots of the quadratic (5.9). Now we can define a coefficient of rolling friction $\mu_{T}$ which is independent of the actual contact angle, a more useful measure of the transverse force arising from a given normal force, by

$$
\mu_{T}=S / \epsilon_{s}^{\prime} T=\epsilon \mu / \epsilon_{s} .
$$

An Atlas programme for the above system of equations has been constructed with $h, f, \gamma, \zeta$ as variable parameters, and run with data in the following ranges:

$$
\gamma=1, \quad 0.5 \leq h \leq 2, \quad 0.5 \leq f \leq 5, \quad 0.1 \leq \xi \leq 10 .
$$

The choice $\gamma=1$, which equates one characteristic time with the semi-contact time, 
ensures that the corresponding creep contribution is effective during contact. Varying $\zeta$ over a wide range from small to large values takes the second characteristic time between comparatively large and comparatively small values, and hence its creep contribution during contact varies over the full range. With a narrow range for the weighting ratio, $h$, the maximum creep contributions associated with the two characteristic times are kept at similar levels. Clearly the viscoelastic memory effects, and resulting nonsymmetry, increase as the maximum total creep $f$ increases, but in view of the present infinitesimal strain assumption it is appropriate that creep and instantaneous (elastic) response remain the same order of magnitude.

The variation of $\Delta$ and $\mu_{T}$ with $\zeta$ is shown in Figs. 2 and 3 for given values of $h$ and $f$ in plots against $\log \zeta$ which is a more natural scaling for the time ratio. Since it is found that changing $h$ within the above range has less significance than changing $f$, the illustrations are restricted to the case $h=1$. When $\zeta=1\left(\gamma_{1}=\gamma_{2}=1\right)$ the two characteristic times coincide and the solution is that for a single-time model with the same total creep $f$, independent of the weighting ratio $h$. It is seen for $h=1$, with both characteristic times equally weighted, that the maximum values of both $\Delta$ and $\mu_{T}$ occur at decreasing values of $\zeta$ as the total creep $f$ increases, and for $f \geq 0.5$ the peaks occur in $\zeta<1$. Thus the nonsymmetry effects from a two-time model, with the second characteristic time in some range of values greater than the scmi-contact time, are greater than those for the single-time (cqual to semi-contact time) model, $\zeta=1$, with the same total creep.

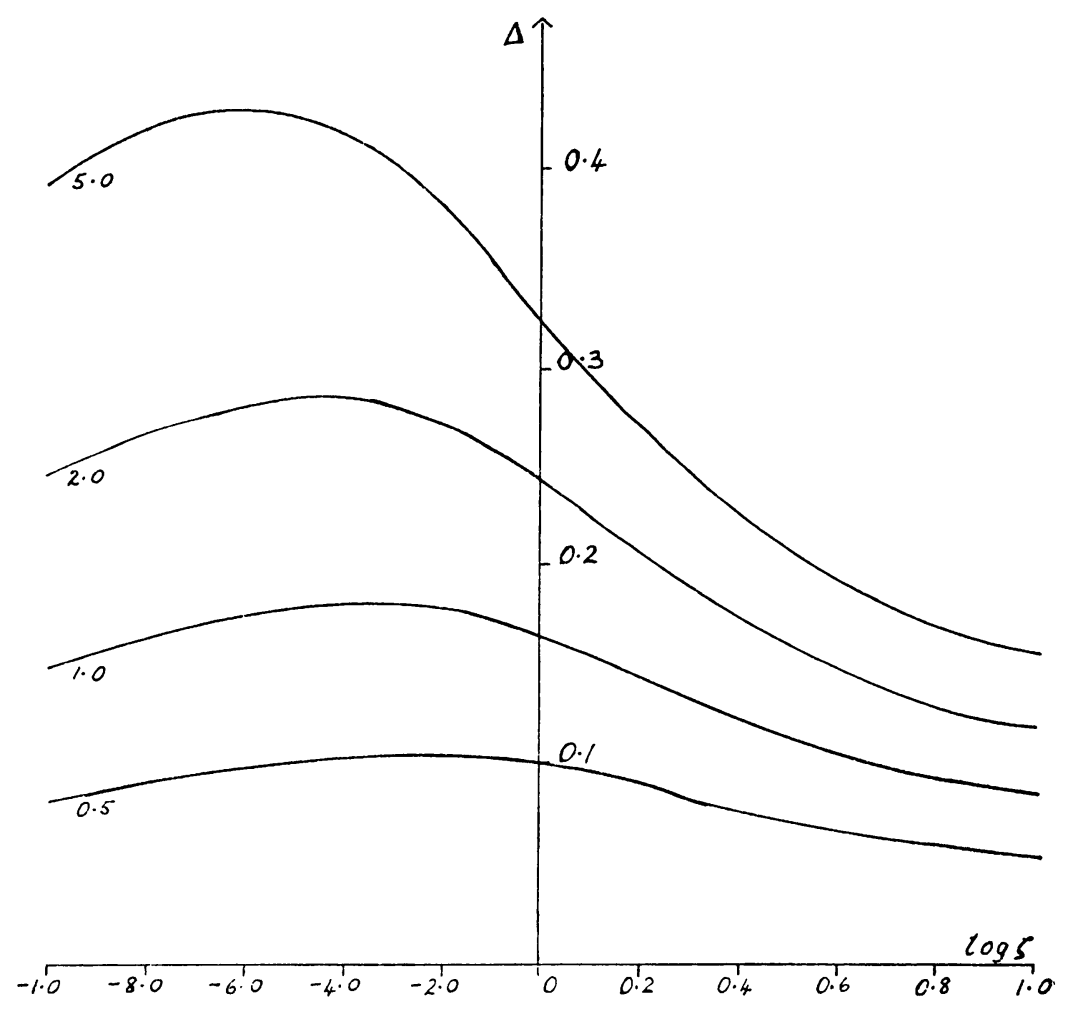

FIg. 2. Variation of $\Delta$ with $\zeta$ for $h=1$ and specified values of $f$. 


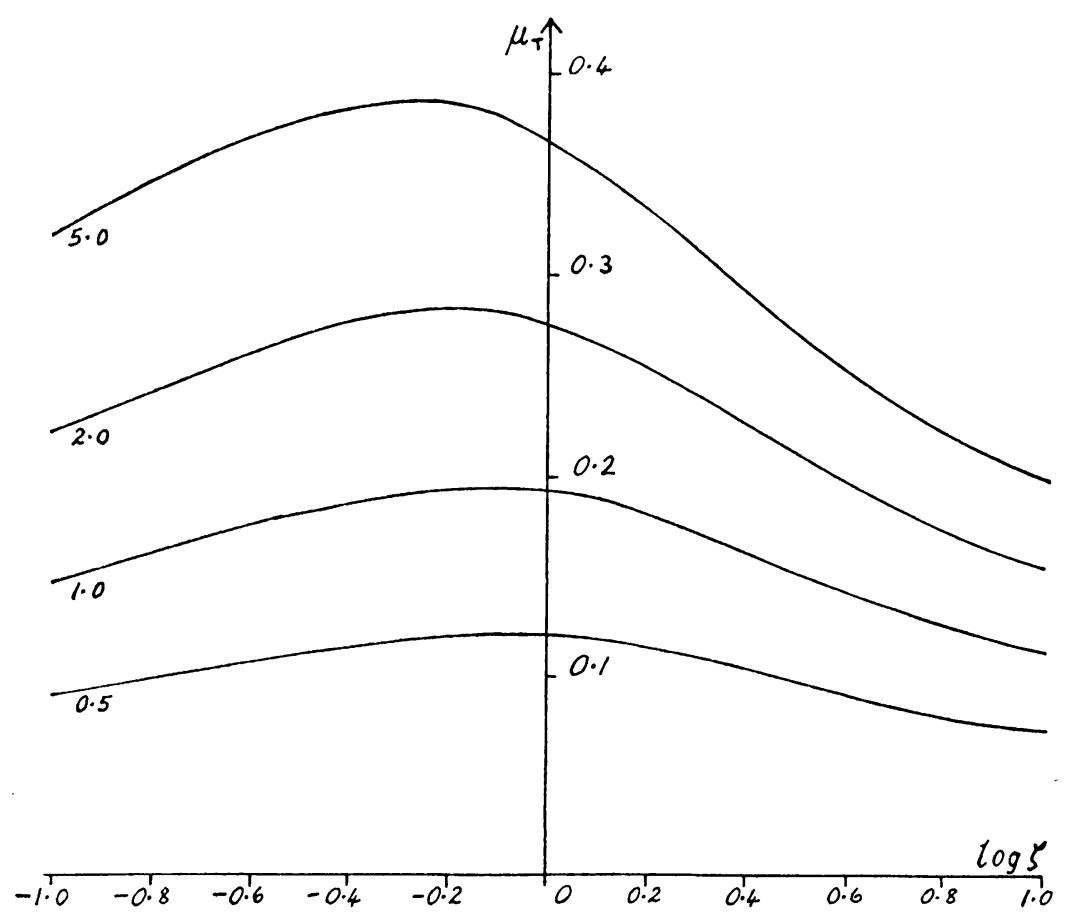

FIg. 3. Variation of $\mu_{T}$ with $\zeta$ for $h=1$ and specified values of $f$.

We can infer that the maximum effect will occur for an overall characteristic time of the material somewhat greater than the semi-contact time, particularly as $f$ increases. The semi-contact angle, as described by $\epsilon / \epsilon_{\mathrm{a}}$, is found in each case to increase monotonically with $\zeta$, and the following table indicates its range of values over $0.1 \leq \zeta \leq 10$ for different values of $f$, with $h=1$.

\begin{tabular}{|c|c|c|c|c|}
\hline$f$ & 0.5 & 1.0 & 2.0 & 5.0 \\
\hline$\epsilon / \epsilon_{s}$ & $0.851-0.935$ & $0.769-0.902$ & $0.680-0.869$ & $0.590-0.838$ \\
\hline
\end{tabular}

Conclusion. A closed form solution has been obtained for rolling contact between dissimilar viscoelastic cylinders, but it should be noted that its construction depends explicitly on the viscoelastic model adopted. That is, the derivation of the integral equation kernel and the crucial differential relations between its parts hinge on the finite spectrum of characteristic times. For a general creep function, or even a continuous spectrum model, the analogous steps do not follow.

\section{REFERENCES}

[1] L. W. Murtand, Exact solutions for rolling contact betueen linear viscoelastic cylinders, Quart. J. Mech. Appl. Math. 20, 73 (1967)

[2] F. G. Tricomi, Integral equations, Interscience, New York, 1957

[3] G. N. Watson, A treatise on the theory of Bessel functions, 2nd ed. Cambridge Univ. Press, Oxford, 1944

[4] S. C. Hunter, The rolling contact of a rigid cylinder with a viscoelastic half space, J. Appl. Mech. 28, 611 (1961) 